\title{
Mapping of QTLs governing agronomic and yield traits in chickpea
}

\author{
S. J. M. Gowda $\cdot$ P. Radhika $\cdot$ L. B. Mhase $\cdot$ \\ B. M. Jamadagni $\cdot$ V. S. Gupta $\cdot$ N. Y. Kadoo
}

Received: 2 January 2010 /Revised: 4 August 2010 /Accepted: 7 August 2010 /Published online: 23 December 2010

(C) Institute of Plant Genetics, Polish Academy of Sciences, Poznan 2010

\begin{abstract}
Chickpea is one of the most important leguminous cool season food crops, cultivated prevalently in South Asia and Middle East. The main objective of this study was to identify quantitative trait loci (QTLs) associated with seven agronomic and yield traits in two recombinant inbred line populations of chickpea derived from the crosses JG62 $\times$ Vijay (JV population) and Vijay $\times$ ICC4958 (VI population) from at least three environments. Single locus QTL analysis involved composite interval mapping (CIM) for individual traits and multiple-trait composite interval mapping (MCIM) for correlated traits to detect pleiotropic QTLs. Two-locus analysis was conducted to identify the main effect QTLs (M-QTLs), epistatic QTLs (E-QTLs) and QTL $\times$ environment interactions. Through CIM analysis, a total of 106 significant QTLs (41 in JV and 65 in VI populations) were identified for the seven traits, of which one QTL each for plant height and days to maturity was common in both the populations. Six pleiotropic QTLs that were consistent over the environments were also identified. LG2 in JV and LG1a in VI
\end{abstract}

S. J. M. Gowda • P. Radhika • V. S. Gupta N. Y. Kadoo $(\bowtie)$ Biochemical Sciences Division, National Chemical Laboratory, Dr. Homi Bhabha Road, Pashan,

Pune 411008, India

e-mail: ny.kadoo@ncl.res.in

L. B. Mhase $\cdot$ B. M. Jamadagni

Pulses Research Station, Mahatma Phule Krishi Vidyapeeth, Rahuri 413722, India

Present Address:

S. J. M. Gowda

State Institute of Plant Breeding, University of Hohenheim, Fruwirthstr. 21,

70599, Stuttgart, Germany contained at least one QTL for each trait. Hence, concentrating on these LGs in molecular breeding programs is most likely to bring simultaneous improvement in these traits.

Keywords Cicer arietinum · QTL analysis · QTL $\times$ environment interaction · Two-locus analysis · Yield traits

\section{Introduction}

Chickpea (Cicer arietinum L.), a cool season grain legume of high nutritive value, is the most important legume in the Indian subcontinent. However, in spite of its importance, its productivity is very low $(\sim 850 \mathrm{~kg} / \mathrm{ha})$ in its intensively cultivated areas. The major causes of low yield of chickpea are: low yield potential and susceptibility of the present day cultivars to various biotic and abiotic stresses and the lack of cultivars' response to intensive management practices like fertilizers, irrigation etc. Identification and mapping of the QTLs governing grain yield and other agronomic traits would increase our understanding of the genetic control of the characters and to use them effectively in breeding programs. The objective of the present study was to perform genetic dissection of seven agronomic and yield traits in chickpea to identify the main effect QTLs (MQTLs) using single locus QTL analysis by composite interval mapping (CIM) and to identify pleiotropic QTLs using multiple-trait composite interval mapping (MCIM). We also conducted two-locus analysis to detect the main effect QTLs (M-QTLs), epistatic QTLs (E-QTLs), QTL × QTL (QQ) and QTL $\times$ environment $(\mathrm{QE}$ and $\mathrm{QQE})$ interactions. 


\section{Materials and methods}

\section{Mapping populations}

Two $\mathrm{F}_{8: 9}$ recombinant inbred line (RIL) populations advanced by single seed descent method from $F_{2}$ to $F_{9}$ were used in the present study. The populations were derived from two intraspecific crosses: JG62 $\times$ Vijay (JV population) and Vijay $\times$ ICC4958 (VI population) and comprised 197 and 108 lines, respectively. The parental genotypes were of desi type of which JG62 is doublepodded and susceptible to fusarium wilt. Vijay is a wilt resistant, drought tolerant variety having high pod number and wider adaptability, whereas ICC4958 is a late wilting, drought tolerant and bold seeded cultivar. The two RIL populations were grown in the field at Pulses Research Station, Mahatma Phule Krishi Vidyapeeth, Rahuri, India.

Evaluation of agronomic and yield traits

The experiment was conducted in randomized block design with two replications during winter seasons of 2002-03, 2003-04, 2004-05 and 2005-06. Sowing was done in $3 \mathrm{~m}$ rows placed $30 \mathrm{~cm}$ apart, accommodating 20 seeds each at $15 \mathrm{~cm}$ distance in a row. Vijay and ICC4958 also served as checks and were sown after every 10 rows of the RILs. The data about seven agronomic and yield traits were recorded on 197 and 108 lines in JV and VI populations, respectively. Ten plants were randomly chosen from each RIL for trait evaluation, excluding the plants at the border or those in border rows. Following seven phenotypic characters were recorded on the selected plants at maturity: (i) plant height (Pht), in cm; (ii) plant spread (Psp), in $\mathrm{cm} \mathrm{-} \mathrm{the} \mathrm{average}$ horizontal spread of the plants; (iii) number of branches per plant (Brp); (iv) number of pods per plant (Pdp); (v) yield per plant (Yld), in g; (vi) 100-seed weight (Swt), in g; and (vii) days to maturity (Dmt). In the VI population, the data were recorded in four environments (environments I: 200203, II: 2003-04, III: 2004-05 and IV: 2005-06), whereas in the JV population, the data were recorded in three environments (environments II: 2003-04, III: 2004-05 and IV: 2005-06). The data from all the environments were also pooled $(\mathrm{P})$ and analyzed in both the populations.

The soil of the field was well-drained, deep and medium black. The average minimum temperature during the duration of crop growth was $14.1 \pm 1.3^{\circ} \mathrm{C}$, while the average maximum temperature was $30.7 \pm 0.2^{\circ} \mathrm{C}$ among the four environments. Similarly, the relative humidity values ranged from $30.5 \pm 2 \%$ to $70.0 \pm 2.5 \%$. The sunshine hours averaged to $8.65 \pm 0.25 \mathrm{hrs}$ during the crop growth period. The number of rainy days ranged from 1 (environment I) to 6 (environment III); however, the amount of rainfall showed a large variation among the four environments and was $1.6 \mathrm{~mm}$ (environment $\mathrm{I}$ ), $38.0 \mathrm{~mm}$ (environment II), $113.8 \mathrm{~mm}$ (environment III) and $47.6 \mathrm{~mm}$ (environment IV).

\section{Statistical analysis}

The phenotypic data analysis was performed using IRRISTAT Ver. 5.0 (IRRI 2005) using "Single site analysis module". The analysis of variance (ANOVA) revealed significant differences among the parental genotypes for all the traits evaluated. The $\mathrm{G} \times \mathrm{E}$ interaction was deciphered using the AMMI (Additive Main effects and Multiplicative Interaction) model in IRRISTAT. Broad sense heritability (\%) was estimated as genotypic variance/phenotypic variance $\left(\sigma_{\mathrm{g}}{ }^{2} / \sigma_{\mathrm{p}}{ }^{2}\right)$. Correlation coefficients among the seven traits were calculated using Qgene Ver. 2.0 (Nelson 1998).

\section{QTL analysis}

The QTLs were identified by single locus QTL analysis through CIM using Windows QTL Cartographer Ver. 2.5 (Wang et al. 2004). The threshold LOD scores were calculated using 1000 permutations (Deorge and Churchill 1996) and a LOD score of 3.0 was used for declaring presence of the QTLs. Model 6 of the CIM was used with forward regression and backward elimination module of Windows QTL Cartographer with scanning intervals of $2.0 \mathrm{cM}$ between the markers. The positional genetic effects and percentage of phenotypic variation of the QTLs were estimated at the significant LOD peaks in the region under consideration. Multiple trait analysis involving MCIM was conducted using the module JZmap QTL available in Windows QTL Cartographer with an objective to detect pleiotropic QTLs. The confidence intervals were obtained by marking positions \pm 1 LOD from the peaks. QTLs in the adjacent intervals and / or with overlapping confidence intervals were treated as a single QTL. Two locus analysis was conducted using QTLNetwork Ver. 2.0 (http://ibi.zju. edu.cn/software/qtlnetwork), where $\mathrm{P}=0.05$ was used as the threshold for detecting M-QTLs or E-QTLs. MapChart Ver. 2.2 (Voorrips 2002) was used to denote the identified QTLs on particular linkage groups.

\section{Results}

Trait variations and correlations

The parental genotypes used to develop the mapping populations were distinct for all the seven agronomic and yield traits (Table 1). These traits showed good fit to the normal distribution in both the populations (data not 
Table 1 Mean values ( \pm S.D.), variation ranges and heritabilities for the agronomic and yield traits in parental lines and the JV and VI mapping populations

\begin{tabular}{|c|c|c|c|c|c|c|c|c|}
\hline \multirow[t]{2}{*}{ Traits } & \multicolumn{3}{|c|}{ Parental lines } & \multicolumn{2}{|c|}{ JV population } & \multicolumn{2}{|c|}{ VI population } & \multirow[t]{2}{*}{ Heritability (\%) } \\
\hline & JG62 & Vijay & ICC4958 & Mean & Range & Mean & Range & \\
\hline Plant height $(\mathrm{cm})$ & $36.9 \pm 4.2$ & $35.1 \pm 4.5$ & $40.6 \pm 4.3$ & $34.1 \pm 2.9$ & $24.3-40.4$ & $37.3 \pm 3.4$ & $30.1-44.9$ & 77 \\
\hline Plant spread (cm) & $18.7 \pm 7.3$ & $22.8 \pm 2.5$ & $24.4 \pm 5.2$ & $22.5 \pm 3.5$ & $13.4-31.5$ & $18.1 \pm 2.0$ & $12.5-23.3$ & 36 \\
\hline Number of branches per plant & $8.3 \pm 4.4$ & $11.2 \pm 1.9$ & $11.5 \pm 2.8$ & $11.2 \pm 2.7$ & $5.5-18.8$ & $10.1 \pm 1.2$ & $6.0-13.5$ & 62 \\
\hline Number of pods per plant & $23.4 \pm 5.6$ & $35.1 \pm 4.6$ & $34.0 \pm 8.7$ & $34.2 \pm 11.9$ & $11.7-87.3$ & $30.0 \pm 5.1$ & $15.1-47.0$ & 64 \\
\hline Yield per plant $(\mathrm{g})$ & $5.6 \pm 2.8$ & $7.8 \pm 1.5$ & $10.8 \pm 4.5$ & $5.8 \pm 2.2$ & $1.2-13.1$ & $7.0 \pm 1.6$ & $3.5-11.0$ & 52 \\
\hline 100-seed weight (g) & $14.7 \pm 0.8$ & $18.0 \pm 0.8$ & $34.4 \pm 2.4$ & $15.6 \pm 2.1$ & $8.5-24.6$ & $24.3 \pm 4.2$ & $17.0-35.5$ & 84 \\
\hline Days to maturity & $103.0 \pm 4.5$ & $106.0 \pm 3.0$ & $117.0 \pm 3.7$ & $108.2 \pm 3.8$ & $99.5-118.0$ & $113.9 \pm 2.9$ & $107.0-121.0$ & 63 \\
\hline
\end{tabular}

shown). Transgressive segregants were observed in all the environments and comparisons between the best parent and the best RIL showed a significant difference for most of the traits (Table 1). ANOVA using the AMMI model was performed using IRRISTAT for all the traits in both the populations. The phenotypic values for RILs in both the populations differed significantly for all the traits (data not shown).

The estimates of broad-sense heritability ranged from $36 \%$ to $84 \%$ (Table 1 ). The highest positive correlation was observed between Brp and Pdp (0.669) in the JV population, whereas in the VI population, Pht and Swt exhibited the highest positive correlation of 0.660 . Yld showed significant positive correlation with Pht, Brp, Pdp and Swt in both the populations, while Dmt was significantly correlated only with Swt in the JV population.

QTLs resolved by single locus analysis

For QTL analysis, two linkage maps for the two populations were independently developed and used. These maps are broadly based on the two maps previously used to develop the integrated intraspecific linkage map of chickpea (Radhika et al. 2007), but additionally have 26 new markers. The current map based on JV population has 123 markers on eight linkage groups and covered $623.2 \mathrm{cM}$ with marker density of $6.1 \mathrm{cM}$. Whereas, the current map based on VI population has 176 markers spanning $688.4 \mathrm{cM}$ with an average of $5.9 \mathrm{cM}$ interval between two markers.

\section{Composite interval mapping in JV population}

In this population, 41 significant QTLs (LOD $\geq 3.0)$ (Table 2; Fig. 1) and 47 suggestive QTLs (2.0 $>$ LOD $<$ 3.0) (data not shown) were identified for the seven traits. The number of significant QTLs for individual traits ranged from two (Swt) to 12 (Psp). Twenty-one significant QTLs were detected in the pooled data followed by environment IV (15 QTLs), while only three QTLs were detected in environment II. At least one QTL for each trait was detected in environment III and the pooled data. The marker TA47 was associated with QTLs for as many as four traits viz. Psp, Brp, Pdp and Yld, while STMS13 was associated with QTLs for three traits viz. Pht, Brp and Dmt. The highest phenotypic variation of $23.2 \%$ was explained by QDmt.ncl-2.1, followed by QBrp.ncl-2.1 (19.3\%). Most of the QTLs were environment specific and only 11 of the 41 QTLs expressed in more than one environment. This was particularly evident in the case of Psp QTLs, where 11 of the 12 Psp QTLs were environment specific. On the contrary, four of the ten Dmt QTLs expressed in more than one environment. LG2 was associated with most of the QTLs (14), wherein the QTLs were clustered in two groups (Fig. 1). LG2 also had at least one QTL for each trait; whereas no QTL could be mapped on LG6.

Vijay exhibited higher phenotypic values than JG62 for all the traits except Pht. For four of the five Pht QTLs, Vijay alleles decreased plant height in the population while the JG62 allele of QPht.ncl-1.2 increased the trait value. Similarly for Psp, Pdp, Yld and Swt QTLs, the alleles from Vijay positively influenced the phenotypic values. On the contrary, JG62 alleles influenced five of the six Dmt QTLs to reduce the days to maturity. Of the five Brp QTLs, only QBrp.ncl-2.1 was positively influenced by Vijay alleles in two environments and increased the number of branches per plant.

Composite interval mapping in VI population

Sixty-five significant QTLs (LOD $\geq 3.0$ ) (Table 3; Fig. 2) and 77 suggestive QTLs $(2.0>\operatorname{LOD}<3.0)$ (data not shown) were detected for the seven traits in this population. The number of significant QTLs for individual traits ranged from five (Yld) to 16 (Psp) and a maximum of 24 QTLs were detected in environment IV followed by the pooled 
Table 2 The QTLs detected through composite interval mapping for the seven agronomic and yield traits in the JV population
${ }^{\text {a }}$ In parentheses, the Roman numerals indicate environments in which the QTL was detected.

${ }^{\mathbf{b}}$ The LOD scores for each environment

${ }^{\mathbf{c}} \mathrm{A}$ : additive effect

d PVE: Phenotypic variation explained

\begin{tabular}{|c|c|c|c|c|c|c|}
\hline Trait & Marker $^{\mathrm{a}}$ & QTL & Position (cM) & $\mathrm{LOD}^{\mathrm{b}}$ & $A^{c}$ & $\operatorname{PVE}^{\mathrm{d}}(\%)$ \\
\hline \multicolumn{7}{|c|}{ Plant height } \\
\hline LG1 & TAA170 (II / P) & QPht.ncl-1.1 & $44.4 / 42.4$ & $2.2 / 3.2$ & $-1.3 /-1.0$ & $9.6 / 10.6$ \\
\hline LG1 & TS46 (III) & QPht.ncl-1.2 & 70.8 & 4.6 & 2.3 & 15.9 \\
\hline LG2 & TA116y (P) & QPht.ncl-2.1 & 55.3 & 3.9 & -1.2 & 12.8 \\
\hline LG2 & STMS13 (P) & QPht.ncl-2.2 & 90.0 & 3.5 & -1.0 & 11.4 \\
\hline LG4 & TR7s (IV) & QPht.ncl-4.1 & 52.8 & 3.8 & -1.9 & 12.3 \\
\hline \multicolumn{7}{|c|}{ Plant spread } \\
\hline LG1 & NCPGR27 (III) & QPsp.ncl-1.1 & 36.4 & 3.5 & 1.8 & 12.6 \\
\hline LG2 & TA47 (III) & QPsp.ncl-2.1 & 46.8 & 3.7 & 1.9 & 12.2 \\
\hline LG3 & TA117 (III) & QPsp.ncl-3.1 & 10.6 & 3.0 & -1.6 & 10.0 \\
\hline LG3 & TA180 (IV) & QPsp.ncl-3.2 & 22.5 & 3.0 & -3.0 & 13.4 \\
\hline LG4 & TA14s (P) & QPsp.ncl-4.1 & 5.0 & 3.0 & -1.1 & 8.1 \\
\hline LG4 & T146 (IV) & QPsp.ncl-4.2 & 15.2 & 3.1 & 3.3 & 10.4 \\
\hline LG4 & GA34 (P) & QPsp.ncl-4.3 & 63.7 & 3.4 & 1.5 & 13.8 \\
\hline LG4 & TR1s (P) & QPsp.ncl-4.4 & 70.2 & 2.9 & 1.4 & 12.4 \\
\hline LG5 & TA96 (P) & QPsp.ncl-5.1 & 38.4 & 3.1 & 1.4 & 11.2 \\
\hline LG5 & TA59 (P) & QPsp.ncl-5.2 & 44.8 & 4.2 & 1.5 & 11.8 \\
\hline LG5 & TS47 (P) & QPsp.ncl-5.3 & 50.3 & 3.1 & 1.3 & 9.8 \\
\hline LG8 & NCPGR98 (IV / P) & QPsp.ncl-8.1 & $0.0 / 0.0$ & $3.0 / 3.0$ & $2.6 / 1.2$ & $10.6 / 8.4$ \\
\hline \multicolumn{7}{|c|}{ Number of branches per plant } \\
\hline LG1 & TAA170 (III) & QBrp.ncl-1.1 & 42.4 & 3.0 & -1.1 & 9.6 \\
\hline LG2 & TA47 (IV / P) & QBrp.ncl-2.1 & $46.8 / 47.0$ & $5.1 / 3.2$ & $3.5 / 1.0$ & $19.3 / 10.6$ \\
\hline LG2 & STMS13 (IV) & QBrp.ncl-2.2 & 92.0 & 3.8 & -2.8 & 17.4 \\
\hline LG3 & TA28 (IV) & QBrp.ncl-3.1 & 0.0 & 4.2 & -2.4 & 11.9 \\
\hline LG3 & TA117 (IV) & QBrp.ncl-3.2 & 10.6 & 3.0 & -2.2 & 9.0 \\
\hline \multicolumn{7}{|c|}{ Number of pods per plant } \\
\hline LG2 & TA47 (IV / P) & QPdp.ncl-2.1 & $46.8 / 46.0$ & $3.5 / 2.4$ & $13.4 / 4.4$ & $12.3 / 8.1$ \\
\hline LG2 & TR60 (III) & QPdp.ncl-2.2 & 55.9 & 3.4 & -6.7 & 11.3 \\
\hline LG2 & TS35 (III) & QPdp.ncl-2.3 & 82.0 & 3.0 & 6.4 & 10.6 \\
\hline LG2 & TR29 (III) & QPdp.ncl-2.4 & 88.6 & 3.9 & 6.8 & 12.7 \\
\hline LG3 & TA28 (IV) & QPdp.ncl-3.1 & 0.0 & 3.6 & -11.2 & 12.0 \\
\hline LG4 & T146 (IV / P) & QPdp.ncl-4.1 & $29.2 / 17.8$ & $2.4 / 3.0$ & $6.4 / 6.4$ & $9.1 / 14.5$ \\
\hline \multicolumn{7}{|c|}{ Yield per plant } \\
\hline LG2 & TA47 (P) & QYld.ncl-2.1 & 46.8 & 3.1 & 1.3 & 10.4 \\
\hline LG2 & TS35 (II) & QYld.ncl-2.2 & 82.0 & 3.0 & 1.6 & 13.0 \\
\hline LG2 & TR29 (II) & QYld.ncl-2.3 & 88.6 & 4.1 & 1.8 & 15.8 \\
\hline LG5 & TA127 (IV / P) & QYld.ncl-5.1 & $113.2 / 113.0$ & $3.4 / 2.0$ & $-1.2 /-2.7$ & $9.7 / 6.7$ \\
\hline LG7 & UBC299y (III) & QYld.ncl-7.1 & 18.2 & 3.1 & 1.7 & 12.1 \\
\hline \multicolumn{7}{|c|}{100 -seed weight } \\
\hline LG2 & UBC238y (III / P) & QSwt.ncl-2.1 & $81.9 / 81.0$ & $3.2 / 2.0$ & $1.3 / 0.7$ & $11.4 / 8.0$ \\
\hline LG4 & TR7s (III) & QSwt.ncl-4.1 & 52.5 & 3.4 & 1.8 & 13.0 \\
\hline \multicolumn{7}{|c|}{ Days to maturity } \\
\hline LG1 & NCPGR80 (IV / P) & QDmt.ncl-1.1 & $26.5 / 26.5$ & $4.3 / 4.8$ & $-2.1 /-1.8$ & $14.1 / 18.7$ \\
\hline LG2 & STMS13 (IV / P) & QDmt.ncl-2.1 & $100.0 / 98.0$ & $3.5 / 4.7$ & $-1.8 /-1.9$ & $11.4 / 23.2$ \\
\hline LG3 & TA117 (III) & QDmt.ncl-3.1 & 10.6 & 3.9 & -2.2 & 14.4 \\
\hline LG3 & TA180 (III / P) & QDmt.ncl-3.2 & $14.5 / 14.5$ & $3.5 / 2.9$ & $-2.2 /-1.3$ & $15.2 / 10.2$ \\
\hline LG7 & UBC299x (IV / P) & QDmt.ncl-7.1 & $30.9 / 36.8$ & $3.5 / 2.0$ & $-1.7 /-1.8$ & $18.0 / 7.3$ \\
\hline LG8 & UBC90y $(\mathrm{P})$ & QDmt.ncl-8.1 & 44.7 & 3.7 & 1.6 & 16.3 \\
\hline
\end{tabular}


Fig. 1 Linkage map depicting QTLs for seven agronomic and yield traits detected in the JV (JG62 $\times$ Vijay) mapping population. The QTLs have been designated using the letter 'Q' followed by the trait name abbreviations (given below): ncl (abbreviation of the organization's name), the linkage group number and the QTL number for the trait. The traits are: plant height (Pht), plant spread (Psp), number of branches per plant (Brp), number of pods per plant (Pdp), grain yield (Yld), 100seed weight (Swt) and days to maturity (Dmt). The traits, seeds per pod $(S p p)$ and double podding (Sfl), which were mapped earlier (Radhika et al. 2007) have been shown in italics. Corresponding LGs of Winter et al. (2000) reference map have been indicated in Roman numerals in parentheses

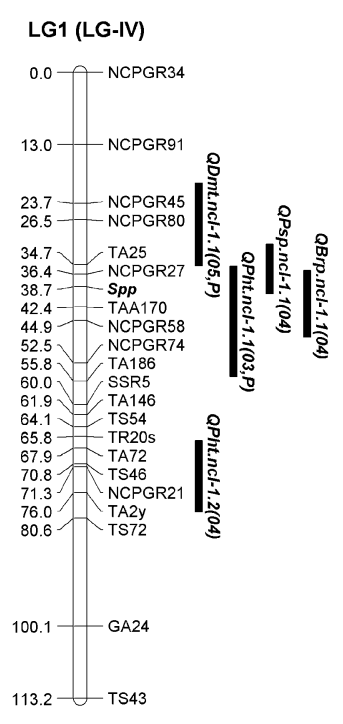

LG2 (LG-III,V)

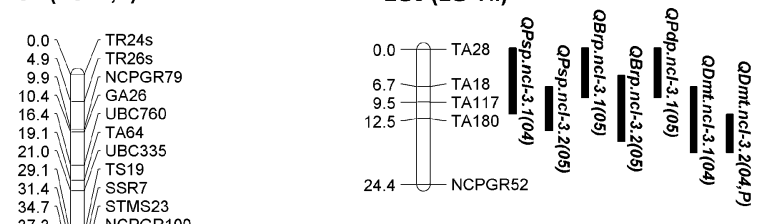

LG3 (LG-VII)

41.

41.0
42.8

$\left.\begin{array}{l}42.8 \\ 43.0\end{array}\right)$. STMS14

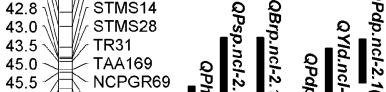

46.8 ) TA47

48.5 TA135s

50.1
51.1

55.8.

57.8 NCPGR63

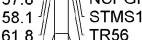

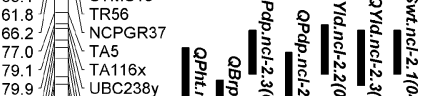

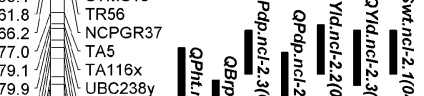

82.0 .

$\left.\left.\begin{array}{l}83.4 \\ 88.6\end{array}\right]\right)$.

$\left.\left.\begin{array}{l}88.6 \\ 90.0\end{array}\right]\right)\left.\right|_{\text {TRTM } 13}$

104.6 NCPGR48
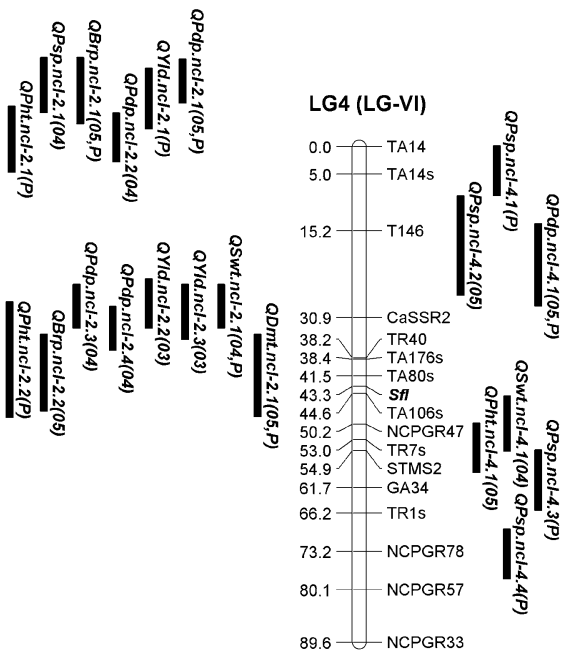

LG5 (LG-II)
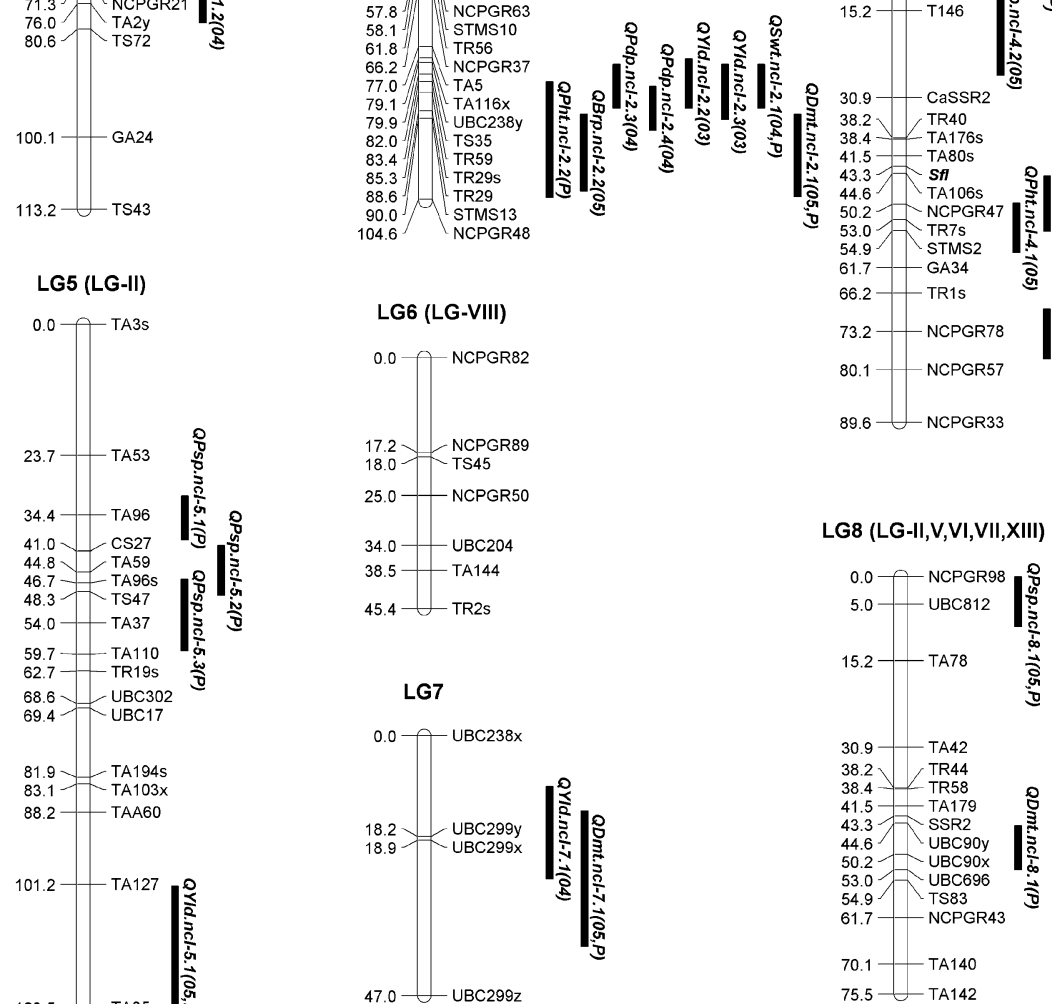

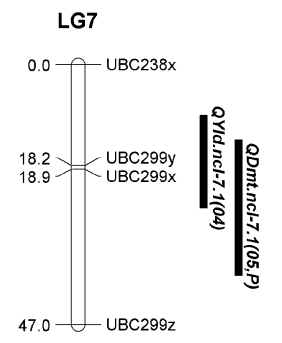

$75.5 \smile$ TA142 data (21 QTLs). Most of the QTLs were environment specific, however 24 QTLs expressed in more than one environment. As observed in the JV population, 12 of the 16 Psp QTLs detected in this population were environment specific and, in contrast, only one of the nine Dmt QTLs expressed in more than one environment. All seven Brp QTLs expressed in individual environments, while 11 of the 14 Swt QTLs were expressed in more than one environment. Only two QTLs (QPht.ncl-1.1 and QDmt.ncl-1.1) were common in the two populations. The highest phenotypic variation of $>29.6 \%$ was exhibited by the QTLs QPdp.ncl-3.2 and QPdp.ncl-4.2. Interestingly, the marker TAA170 was associated with QTLs for many traits, viz. Pht and Brp in JV population and Pht, Yld and Swt in VI population. LG1a in the VI map contained the most number of QTLs (27) and had at least one QTL for each trait, while only two QTLs could be mapped on LG6.
ICC4958 exhibited higher phenotypic values than Vijay for all the traits except Pdp (Table 1). For 48 of the 65 QTLs, the alleles from Vijay increased phenotypic values for the respective traits. Particularly, all the Pht and Yld QTLs and 13 of the 14 Swt QTLs were positively influenced by Vijay alleles. However, in case of Dmt QTLs, alleles from both the parents contributed equally to the phenotype. Among the total of 106 significant QTLs identified in the two populations, Vijay alleles influenced 73 QTLs (25 in JV and 48 in VI population), whereas 16 QTLs in JV and 17 QTLs in VI population had trait enhancing alleles from JG62 and ICC4958, respectively.

\section{Multiple-trait Composite Interval Mapping (MCIM)}

Single locus MCIM was conducted in both the populations (data not shown). All the QTLs detected through single 
Table 3 The QTLs detected through composite interval mapping for the seven agronomic and yield traits in the VI population

\begin{tabular}{|c|c|c|c|c|c|c|}
\hline Trait & Marker $^{\mathrm{a}}$ & QTL & Position (cM) & $\mathrm{LOD}^{\mathrm{b}}$ & $\mathrm{A}^{\mathrm{c}}$ & $\operatorname{PVE}^{\mathrm{d}}(\%)$ \\
\hline \multicolumn{7}{|c|}{ Plant height } \\
\hline LG1a & QPht.ncl-1.3 & TR20s (III / IV / P) & $45.8 / 45.8 / 45.7$ & $2.7 / 4.6 / 3.9$ & $-1.6 /-2.5 /-1.3$ & $8.1 / 13.8 / 11.0$ \\
\hline LG1a & QPht.ncl-1.4 & TS54 (I) & 55.4 & 3.2 & -2.0 & 11.0 \\
\hline LG1a & QPht.ncl-1.5 & RGA6y (P) & 71.2 & 3.0 & -1.2 & 10.9 \\
\hline LG1a & QPht.ncl-1.1 & TAA170 (I / III) & $83.5 / 83.5$ & $3.5 / 3.1$ & $-1.9 /-1.5$ & $10.1 / 10.6$ \\
\hline LG4 & QPht.ncl-4.2 & NCPGR57 (III) & 39.8 & 3.2 & -2.0 & 16.0 \\
\hline LG5 & QPht.ncl-5.1 & TS12 (I) & 26.4 & 3.0 & -1.6 & 7.2 \\
\hline LG7 & QPht.ncl-7.1 & UBC77y (III) & 68.6 & 3.6 & -1.6 & 11.3 \\
\hline \multicolumn{7}{|c|}{ Plant spread } \\
\hline LG1a & QPsp.ncl-1.2 & UBC43z (IV / P) & $28.4 / 28.4$ & $3.2 / 2.0$ & $2.7 / 0.6$ & $7.5 / 5.2$ \\
\hline LG1a & QPsp.ncl-1.3 & TR20s (I) & 45.8 & 3.0 & -0.8 & 9.6 \\
\hline LG1a & QPsp.ncl-1.4 & TS72 (IV / P) & $49.2 / 49.2$ & $3.2 / 2.0$ & $3.1 / 0.7$ & $10.9 / 7.4$ \\
\hline LG1a & QPsp.ncl-1.5 & H1A12 (IV) & 75.9 & 3.0 & 2.7 & 8.4 \\
\hline LG1a & QPsp.ncl-1.6 & UBC840 (IV) & 88.3 & 3.9 & -2.8 & 9.6 \\
\hline LG2 & QPsp.ncl-2.2 & UBC467 (III) & 0.0 & 3.2 & -0.9 & 8.8 \\
\hline LG2 & QPsp.ncl-2.3 & TR29 (I) & 72.9 & 4.6 & 1.0 & 15.8 \\
\hline LG2 & QPsp.ncl-2.4 & H1B02 (I) & 87.8 & 4.4 & -1.0 & 14.8 \\
\hline LG2 & QPsp.ncl-2.5 & NCPGR65 (III) & 100.8 & 4.4 & -1.1 & 13.7 \\
\hline LG4 & QPsp.ncl-4.5 & NCPGR78 (IV) & 14.7 & 4.7 & -3.8 & 16.3 \\
\hline LG4 & QPsp.ncl-4.6 & TA176s (IV) & 20.5 & 5.1 & -4.1 & 15.9 \\
\hline LG4 & QPsp.ncl-4.7 & H1D22/1 (P) & 22.1 & 3.7 & -1.0 & 11.6 \\
\hline LG4 & QPsp.ncl-4.8 & H4G01 (IV) & 22.6 & 5.9 & -4.3 & 16.2 \\
\hline LG4 & QPsp.ncl-4.9 & H3A05/2 (III / IV) & $33.6 / 33.6$ & $2.4 / 4.5$ & $1.1 / 3.7$ & $11.1 / 11.4$ \\
\hline LG6 & QPsp.ncl-6.1 & H1E23y (IV / P) & $2.0 / 4.0$ & $2.9 / 4.2$ & $2.5 / 1.1$ & $8.4 / 20.2$ \\
\hline LG7 & QPsp.ncl-7.1 & UBC43x (III) & 16.0 & 3.5 & 1.1 & 11.9 \\
\hline \multicolumn{7}{|c|}{ Number of branches per plant } \\
\hline LG1a & QBrp.ncl-1.2 & TA2y (I) & 79.2 & 7.0 & 1.0 & 21.9 \\
\hline LG2 & QBrp.ncl-2.3 & SSR8y (I) & 24.7 & 4.5 & 0.7 & 12.9 \\
\hline LG3 & QBrp.ncl-3.3 & UBC810 (II) & 29.0 & 3.1 & -0.7 & 11.8 \\
\hline LG3 & QBrp.ncl-3.4 & H1N12 (P) & 30.8 & 3.1 & -0.4 & 9.3 \\
\hline LG3 & QBrp.ncl-3.5 & NCPGR52 (P) & 83.1 & 3.2 & -0.4 & 9.3 \\
\hline LG4 & QBrp.ncl-4.1 & TA176s (IV) & 18.5 & 3.4 & -1.4 & 9.8 \\
\hline LG6 & QBrp.ncl-6.1 & UBC891 (II) & 49.8 & 4.6 & -0.8 & 12.7 \\
\hline \multicolumn{7}{|c|}{ Number of pods per plant } \\
\hline LG1a & QPdp.ncl-1.1 & TA2y (I) & 79.2 & 5.3 & 4.2 & 16.2 \\
\hline LG1a & QPdp.ncl-1.2 & Spp (I / IV) & $105.2 / 105.0$ & $4.6 / 3.4$ & $2.4 /-6.6$ & $12.4 / 10.0$ \\
\hline LG1a & QPdp.ncl-1.3 & H3A04 (IV) & 123.1 & 4.1 & -7.3 & 12.5 \\
\hline LG3 & QPdp.ncl-3.2 & UBC165 (II / III) & $22.0 / 19.0$ & $7.7 / 3.5$ & $-3.7 / 4.3$ & 29.6/11.2 \\
\hline LG4 & QPdp.ncl-4.2 & TR7s (P) & 4.0 & 6.2 & -3.7 & 29.7 \\
\hline LG4 & QPdp.ncl-4.3 & NCPGR78 (III) & 14.7 & 3.1 & 5.3 & 12.2 \\
\hline LG5 & QPdp.ncl-5.1 & UBC859x (II) & 12.2 & 4.0 & -2.4 & 11.6 \\
\hline LG7 & QPdp.ncl-7.1 & TR2s (II / IV) & $6.0 / 6.0$ & $2.6 / 3.0$ & $-2.3 /-6.0$ & $10.8 / 8.6$ \\
\hline \multicolumn{7}{|c|}{ Yield per plant } \\
\hline LG1a & QYld.ncl-1.1 & TAA170 (I) & 83.5 & 6.0 & -1.0 & 16.6 \\
\hline LG1a & QYld.ncl-1.2 & STMS21x (P) & 89.9 & 4.8 & -0.7 & 15.5 \\
\hline LG1a & QYld.ncl-1.3 & Spp (IV / P) & $105.3 / 105.0$ & $3.0 / 2.5$ & $-1.4 /-0.5$ & $8.6 / 6.5$ \\
\hline LG3 & QYld.ncl-3.1 & NCPGR52 (II) & 83.1 & 3.5 & -1.0 & 11.5 \\
\hline LG5 & QYld.ncl-5.2 & TA103x (I / II) & $56.0 / 56.0$ & $4.6 / 2.0$ & $-0.8 /-0.7$ & $11.4 / 6.1$ \\
\hline
\end{tabular}


Table 3 (continued)

\begin{tabular}{|c|c|c|c|c|c|c|}
\hline Trait & Marker $^{\mathrm{a}}$ & QTL & Position (cM) & $\mathrm{LOD}^{\mathrm{b}}$ & $\mathrm{A}^{\mathrm{c}}$ & $\operatorname{PVE}^{\mathrm{d}}(\%)$ \\
\hline \multicolumn{7}{|c|}{100 -seed weight } \\
\hline LG1a & QSwt.ncl-1.1 & UBC706 (I) & 62.8 & 6.8 & -2.0 & 16.9 \\
\hline LG1a & QSwt.ncl-1.2 & H4G11 (IV / P) & $64.2 / 64.2$ & $3.3 / 2.2$ & $-2.1 /-1.2$ & $10.3 / 5.3$ \\
\hline LG1a & QSwt.ncl-1.3 & NCPGR74 (P) & 68.3 & 3.0 & -1.3 & 6.8 \\
\hline LG1a & QSwt.ncl-1.4 & RGA6y (III / P) & $71.2 / 71.2$ & $5.2 / 5.8$ & $-2.4 /-2.2$ & $16.8 / 18.7$ \\
\hline LG1a & QSwt.ncl-1.5 & H1G22 (I / II) & $73.7 / 73.0$ & $3.2 / 2.9$ & $-1.4 /-2.7$ & $8.9 / 12.0$ \\
\hline LG1a & QSwt.ncl-1.6 & H1A12 (I / P) & $75.9 / 75.0$ & $3.3 / 3.0$ & $-1.4 /-1.4$ & $8.9 / 8.5$ \\
\hline LG1a & QSwt.ncl-1.7 & TAA170 (I / II / III / P) & $83.5 / 83.0 / 82.8 / 83.0$ & $4.9 / 5.7 / 3.9 / 5.2$ & $-1.7 /-3.8 /-2.2 /-2.1$ & $15.7 / 24.0 / 13.7 / 18.2$ \\
\hline LG1a & QSwt.ncl-1.8 & STMS21x (I) & 89.9 & 3.7 & -1.4 & 10.0 \\
\hline LG2 & QSwt.ncl-2.2 & SSR8y (II / III) & $24.7 / 25.0$ & $2.6 / 3.1$ & $-2.1 /-1.5$ & $6.4 / 7.2$ \\
\hline LG2 & QSwt.ncl-2.3 & TR33 (I) & 27.6 & 5.2 & 1.6 & 11.8 \\
\hline LG3 & QSwt.ncl-3.1 & TA78 (III / P) & $73.6 / 73.0$ & $2.8 / 3.9$ & $-1.4 /-1.3$ & $6.2 / 8.0$ \\
\hline LG7 & QSwt.ncl-7.1 & UBC190y (I) & 90.3 & 4.9 & -2.1 & 20.5 \\
\hline LG7 & QSwt.ncl-7.2 & UBC249z (I / IV / P) & $110.2 / 110.0 / 110.2$ & $7.4 / 3.1 / 4.3$ & $-2.1 /-2.1 /-1.8$ & 19.1/10.1/11.3 \\
\hline LG7 & QSwt.ncl-7.3 & UBC249y (IV) & 114.9 & 3.1 & -2.2 & 10.6 \\
\hline \multicolumn{7}{|c|}{ Days to maturity } \\
\hline LG1a & QDmt.ncl-1.2 & TS54 (IV) & 57.4 & 4.6 & -1.8 & 17.5 \\
\hline LG1a & QDmt.ncl-1.3 & UBC346 (P) & 117.7 & 5.1 & -1.1 & 12.9 \\
\hline LG1b & QDmt.ncl-1.1 & NCPGR80 (IV) & 12.0 & 3.7 & 1.7 & 13.9 \\
\hline LG2 & QDmt.ncl-2.2 & TA42 (P) & 52.6 & 3.2 & -0.9 & 9.1 \\
\hline LG2 & QDmt.ncl-2.3 & UBC881 (IV) & 59.1 & 3.0 & -1.8 & 14.8 \\
\hline LG4 & QDmt.ncl-4.1 & UBC284 (IV) & 32.9 & 3.9 & 1.5 & 10.2 \\
\hline LG5 & QDmt.ncl-5.1 & TS12 (IV / P) & $26.4 / 26.4$ & $2.2 / 3.1$ & $1.1 / 0.9$ & $6.0 / 9.1$ \\
\hline LG7 & QDmt.ncl-7.2 & UBC77y (IV) & 68.6 & 3.2 & 1.3 & 8.1 \\
\hline
\end{tabular}

\footnotetext{
${ }^{a}$ In parentheses, the Roman numerals indicate environments in which the QTL was detected.

b The LOD scores for each environment

${ }^{\mathbf{c}} \mathrm{A}$ : additive effect

d PVE: Phenotypic variation explained
}

locus MCIM were also observed in joint MCIM. In the JV population, at least one QTL for each trait was detected using single locus MCIM and 37 QTLs were detected using joint MCIM. Similarly in the VI population, 20 QTLs (one QTL each for Pdp and Yld; two QTLs for Brp, three QTLs each for Pht, Psp, Dmt and seven QTLs for Swt) were detected using single locus MCIM and 48 QTLs were detected using joint MCIM. Four QTLs in JV and three QTLs in VI population were pleiotropic (Table 4).

QTLs resolved by two-locus analysis

Two-locus QTL analysis was performed in both the populations and the results are summarized in Tables 5 and 6. Seventeen QTLs (5 M-QTLs and 12 E-QTLs) in the JV population and 29 QTLs (9 M-QTLs and 20 E-QTLs) in the VI population that were involved in $\mathrm{QE}$ and $\mathrm{QQE}$ interactions were detected. Two of the five M-QTLs in JV and seven of the nine M-QTLs in the VI population identified through single locus CIM analysis corresponded to the same and / or adjacent marker intervals (Tables 5 and 6). Four M-QTLs (QBrp.ncl-2.2 and QYld. $n c l-8.1$ in JV population and QPsp.ncl-1.7 and QPdp.ncl5.2 in VI population) exhibited QE interactions. In the VI population, two additional QTLs (for Brp and Yld) had no main effects but were involved in QE interactions. In the JV population, QE interactions were observed for only two traits (Brp and Yld) in all the environments, whereas in the VI population four traits (Psp, Brp, Pdp and Yld) exhibited QE interactions in three environments and only one M-QTL (Q.Pht.ncl-1.3) for plant height was involved in epistatic QQ interactions (Table 5).

The epistatic analysis revealed seven QQ interactions involving 14 QTLs in the JV population and 10 QQ interactions involving 21 QTLs in the VI population (Table 6). Eight QTLs involved in these interactions were also detected in CIM (one QTL for Brp in JV and three QTLs for Psp, two QTLs each for Pht and Dmt in the VI 

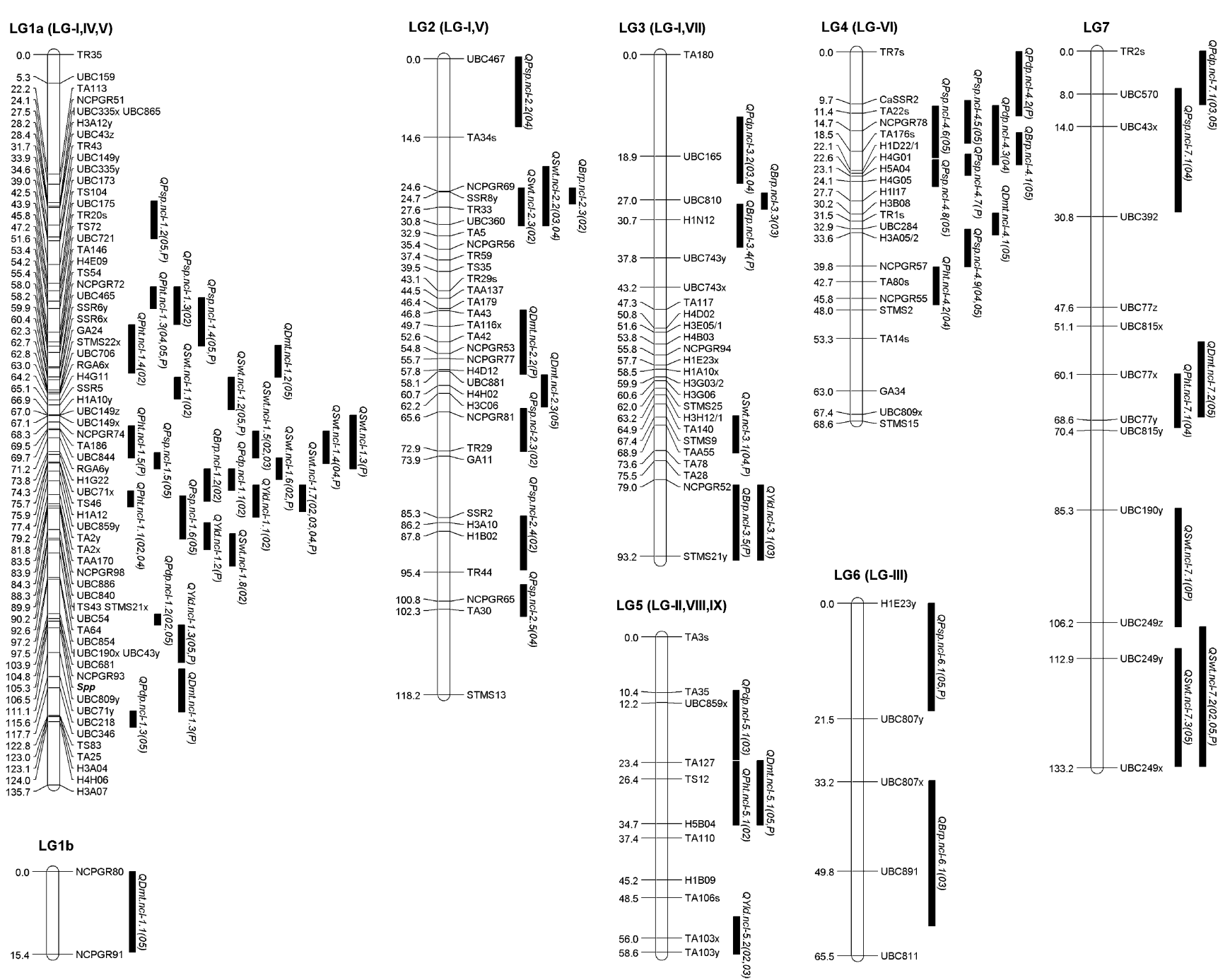

Fig. 1. Corresponding LGs of Winter et al. (2000) reference map have

Fig. 2 Linkage map depicting QTLs for seven agronomic and yield traits detected in the VI (Vijay $\times$ ICC4958) mapping population. For trait description and QTL nomenclature, please see the legend to been indicated in Roman numerals in parentheses

population) (Table 5). In the JV population, no interactions were detected for the traits Yld, Swt and Dmt, whereas in the VI population, QQ interactions were observed for all the traits except Brp. Three QQE interactions were observed for 12 QTLs in the JV population and all these 12 QTLs were E-QTLs involving

four traits, two QTLs each for Pht and Psp, four QTLs each for Brp and Pdp. In the VI population, 18 QTLs were involved in four QQE interactions of which 16 were EQTLs involving three traits (Psp, Pdp and Dmt) and the remaining two QTLs (for Yld) were involved only in QQE interactions. The QTLs for Brp in JV, Pht and Swt in the

Table 4 Pleiotropic

QTLs detected for the seven agronomic and yield traits in JV and VI populations. The trait abbreviations are: plant height (Pht), number of branches per plant (Brp), number of pods per plant (Pdp), plant spread (Psp), 100 -seed weight (Swt) and grain yield (Yld)

\begin{tabular}{lllll}
\hline Traits & LG & Marker interval & Position & Population \\
\hline Pht+Brp & LG2 & NCPGR100-TR31 & $37.0-42.8$ & JV \\
Brp+Pdp & LG2 & TA47-TA135s & $46.0-48.5$ & JV \\
Brp+Psp+Pdp+Swt & LG2 & TA5-TA116x & $77.1-79.1$ & JV \\
Psp+Pdp+Yld & LG2 & TR29-STMS13 & $88.6-90.1$ & JV \\
Pht+Psp & LG1a & TR43-UBC149y & $31.6-33.9$ & VI \\
Pht+Yld+Swt & LG1a & TA2x-TAA170 & $81.8-83.5$ & VI \\
Pdp+Yld & LG5 & TA103x-TA103y & $56.0-58.6$ & VI \\
\hline
\end{tabular}


Table 5 QTLs with main effects and those involved in interaction with the environment for seven agronomic and yield traits in chickpea detected in JV and VI populations following two-locus analysis

\begin{tabular}{|c|c|c|c|c|}
\hline Trait/Marker interval $^{\mathrm{a}}$ & QTL & LG (position) & $\mathrm{A}^{\mathrm{b}}$ & $\mathrm{AE}^{\mathrm{b}}$ \\
\hline \multicolumn{5}{|l|}{ Plant height } \\
\hline TR20s-TS72 (VI, 3) & QPht.ncl-1.3 & LG1a (45.8) & -0.97 & - \\
\hline \multicolumn{5}{|l|}{ Plant spread } \\
\hline NCPGR98-UBC812 (JV, 2) & QPsp.ncl-8.1 & LG8 $(0.0)$ & -1.30 & - \\
\hline UBC43z-TR43 (VI, 3) & QPsp.ncl-1.7 & LG1a (28.4) & 0.83 & $1.83[\mathrm{AE}(\mathrm{IV})]$ \\
\hline \multicolumn{5}{|l|}{ Number of branches per plant } \\
\hline TAA170-NCPGR98 (VI, 3) & QBrp.ncl-1.6 & LG1a (83.5) & - & $0.96[\mathrm{AE}(\mathrm{IV})]$ \\
\hline STMS13-NCPGR48 (JV, 7) & QBrp.ncl-2.2 & LG2 (90.0) & 0.63 & $-0.70[\mathrm{AE}(\mathrm{I})],-0.73[\mathrm{AE}(\mathrm{II})], 1.48[\mathrm{AE}(\mathrm{III})]$ \\
\hline \multicolumn{5}{|l|}{ Number of pods per plant } \\
\hline TA103x-TA103y (VI, 5) & QPdp.ncl-5.2 & LG5 (58.0) & 1.80 & $-3.40[\mathrm{AE}(\mathrm{I})],-2.51[\mathrm{AE}(\mathrm{II})], 5.93[\mathrm{AE}(\mathrm{IV})]$ \\
\hline \multicolumn{5}{|l|}{ Yield per plant } \\
\hline UBC696-TS83 (JV, 3) & QYld.ncl-8.1 & LG8 (53.0) & 0.87 & $-1.10[\mathrm{AE}(\mathrm{II})], 1.86[\mathrm{AE}(\mathrm{III})]$ \\
\hline TA103x-TA103y (VI, 5) & QYld.ncl-5.2 & LG5 (58.0) & - & $-0.83[\mathrm{AE}(\mathrm{I})], 1.18[\mathrm{AE}(\mathrm{IV})]$ \\
\hline \multicolumn{5}{|l|}{ 100-seed weight } \\
\hline STMS10-TR56 (JV, 5) & QSwt.ncl-2.1 & LG2 (61.1) & -0.90 & - \\
\hline RGA6y-C1G22 (VI, 3) & QSwt.ncl-1.7 & LG1a (73.2) & -1.14 & - \\
\hline TA2x-TAA170 (VI, 3) & QSwt.ncl-1.4 & LG1a (82.8) & -1.90 & - \\
\hline UBC249z-UBC249y (VI, 16) & QSwt.ncl-7.2 & LG7 (111.2) & -1.70 & - \\
\hline \multicolumn{5}{|l|}{ Days to maturity } \\
\hline NCPGR80-TA25 (JV, 7) & QDmt.ncl-1.1 & LG1 (30.5) & 1.88 & - \\
\hline UBC346-TS83 (VI, 6) & QDmt.ncl-1.2 & LG1a (119.8) & -1.39 & - \\
\hline
\end{tabular}

${ }^{\text {a }}$ In parentheses are population name (JV/VI), followed by marker intervals in Arabic numerals.

${ }^{\mathbf{b}} \mathrm{A}$ : additive effect; $\mathrm{AE}(\mathrm{I}), \mathrm{AE}(\mathrm{II}), \mathrm{AE}(\mathrm{III})$ and $\mathrm{AE}(\mathrm{IV})$ : QTL $\times$ environment interaction effects for environments I, II, III and IV, respectively

VI population exhibited only QQ interactions, however, no QQE interactions were observed.

\section{Discussion}

In the present study, we identified QTLs for seven agronomic and yield traits in chickpea using two mapping populations and four environments. We initially performed CIM and MCIM to detect the QTLs using QTL Cartographer and later analyzed them for epistatic interactions using QTLNetwork. All the traits showed good fit to the normal distribution in both the populations, suggesting the absence of epistatic effects among the QTLs. Both the populations showed transgressive segregants for all traits, suggesting the possibility of identifying positive alleles in the superior parent and the negative alleles in the inferior parent.

The broad-sense heritability estimates for the seven traits ranged from $36 \%$ (Psp) to $84 \%$ (Swt). These values agree with those reported earlier in chickpea (Eser 1976; Muehlbauer and Singh 1987; Singh 1991; Abbo et al. 2005; Cobos et al. 2007). Eser (1976) observed 13\% narrow-sense heritability for single plant yield, $25 \%$ for number of pods per plant, $30 \%$ for plant height and $78 \%$ for seed weight in chickpea. The author also concluded that seed weight was the least environmentally influenced trait. Singh (1991) estimated heritability values for several traits viz. seed yield, plant height, number of pods per plant, days to flowering, days to maturity and 100-seed weight to range from $49 \%$ to $91 \%$. Abbo et al. (2005) and Cobos et al. (2007) also reported high heritability values for seed weight (71\%) and seed size (90\%) respectively. In the present study, we also observed high heritability (84\%) for seed weight, followed by plant height (77\%).

\section{Single locus analysis}

Forty-one and 65 significant QTLs (LOD $\geq 3.0)$ were identified and mapped in the JV and VI populations, respectively. For each of the seven traits, the alleles with main effects to increase the phenotypic trait values were observed in either of the parents providing supportive evidence for transgressive segregation observed in the phenotypes. Among the 12 Pht QTLs (five in JV and seven in VI population) only one QTL (QPht.ncl-1.1) was 
Table 6 QTLs interactions involving $(\mathrm{Q} \times \mathrm{Q}$ or $\mathrm{Q} \times \mathrm{Q} \times \mathrm{E})$ for agronomic and yield traits in chickpea detected in JV and VI populations by following two-locus analysis

\begin{tabular}{|c|c|c|c|c|c|c|c|}
\hline Trait/ Marker interval $^{\mathrm{a}}$ & QTL & LG (position) & Trait/Marker interval $^{\mathrm{a}}$ & QTL & LG (position) & $\mathrm{AA}^{\mathrm{b}}$ & $\mathrm{AAE}^{\mathrm{b}}$ \\
\hline \multicolumn{8}{|l|}{ Plant height } \\
\hline TS72-GA24R (JV, 12) & QPht.ncl-1.3 & LG1 (83.6) & TA144-TR2s (JV, 5) & QPht.ncl-6.1 & LG6 (38.5) & -1.22 & $-1.08[\mathrm{AAE}(\mathrm{III})]$ \\
\hline TS35-TR29s (VI, 5) & QPht.ncl-1.7 & LG1a (42.5) & UBC859x-TA127 (VI, 8) & QPht.ncl-5.1 & LG5 (12.2) & 1.18 & - \\
\hline \multicolumn{8}{|l|}{ Plant spread } \\
\hline NCPGR81-TA116y (JV, 2) & QPsp.ncl-2.2 & LG2 (50) & TR56-NCPGR37 (JV, 8) & QPsp.ncl-2.3 & LG2 (65.8) & -1.48 & $1.3[\mathrm{AAE}(\mathrm{II})],-1.9$ [AAE(III)] \\
\hline UBC721-TA146 (VI, 3) & QPsp.ncl-1.6 & LG1a (51.6) & TA117-C4D02 (VI, 4) & QPsp.ncl-3.2 & LG3 (50.3) & 0.8 & $-0.87[\mathrm{AAE}(\mathrm{I})], 1.54[\mathrm{AAE}(\mathrm{IV})]$ \\
\hline TS43-STMS21x (VI, 1) & QPsp.ncl-1.7 & LG1a (89.5) & TR44-NCPGR65 (VI, 4) & QPsp.ncl-2.5 & LG2 (95.4) & -0.85 & $-1.57[\mathrm{AAE}(\mathrm{IV})]$ \\
\hline UBC71y-UBC218 (VI, 4) & QPsp.ncl-1.8 & LG1a (114.2) & UBC743y-TA117 (VI, 7) & QPsp.ncl-3.3 & LG3 (43.2) & -1.27 & $0.53[\mathrm{AAE}(\mathrm{I})], 1.03[\mathrm{AAE}(\mathrm{II})],-2.99[\mathrm{AAE}(\mathrm{IV})]$ \\
\hline TA34s-NCPGR69 (VI, 8) & QPsp.ncl-2.6 & LG2 (14.6) & H3H12/1-TA140 (VI, 4) & QPsp.ncl-3.4 & LG3 (64) & 0.56 & $-0.93[\mathrm{AAE}(\mathrm{I})], 1.73[\mathrm{AAE}(\mathrm{IV})]$ \\
\hline \multicolumn{8}{|l|}{ Number of branches per plant } \\
\hline NCPGR79-GA26 (JV, 3) & QBrp.ncl-2.4 & LG2 (9.0) & TA89-TA116y (JV, 4) & QBrp.ncl-2.3 & LG2 (51) & 0.91 & - \\
\hline UBC17-TA194s (JV, 6) & QBrp.ncl-5.1 & LG5 (70.4) & UBC90y-UBC90x (JV, 3) & QBrp.ncl-8.1 & LG8 (44.6) & -1.18 & $0.79[\mathrm{AAE}(\mathrm{II})],-1.36[\mathrm{AAE}(\mathrm{III})]$ \\
\hline TS45-NCPGR50 (JV, 2) & QBrp.ncl-6.1 & LG6 (18.0) & TA140-TA142 (JV, 3) & QBrp.ncl-8.2 & LG8 (75.1) & 1.05 & $-0.97[\mathrm{AAE}(\mathrm{II})], 1.49[\mathrm{AAE}(\mathrm{III})]$ \\
\hline \multicolumn{8}{|l|}{ Number of pods per plant } \\
\hline UBC760-TA64 (JV, 3) & QPdp.ncl-2.4 & LG2 (17.4) & TA37-TA110 (JV, 4) & QPdp.ncl-5.1 & LG5 (59.0) & -7.85 & $7.1[\mathrm{AAE}(\mathrm{I})],-8.1[\mathrm{AAE}(\mathrm{III})]$ \\
\hline TA186-SSR5 (JV, 5) & QPdp.ncl-1.1 & LG1a (57.8) & UBC17-TA194s (JV, 9) & QPdp.ncl-5.2 & LG5 (72.4) & 4.37 & $-6.13[\mathrm{AAE}(\mathrm{II})], 9.4[\mathrm{AAE}(\mathrm{III})]$ \\
\hline UBC721-TA146 (VI, 3) & QPdp.ncl-1.4 & LG1a (51.6) & H1A10x-H3GO3/2 (VI, 4) & QPdp.ncl-3.3 & LG3 (58.5) & 1.88 & $3.1[\mathrm{AAE}(\mathrm{IV})]$ \\
\hline TA43-TA116x (VI, 2) & QPdp.ncl-2.3 & LG2 (46.8) & UBC190y-UBC249z (VI, 13) & QPdp.ncl-7.3 & LG7 (95.3) & 3.6 & -3.87 [AAE(II)], -3.5 [AAE(III)], 8.1 [AAE(IV)] \\
\hline TA116x-TA42 (VI, 4) & $Q P d p . n c l-2.4$ & LG2 (50.7) & UBC249y-UBC249x (VI, 12) & QPdp.ncl-7.4 & LG7 (112.9) & -1.39 & $4.1[\mathrm{AAE}(\mathrm{III})],-6.2[\mathrm{AAE}(\mathrm{IV})]$ \\
\hline \multicolumn{8}{|l|}{ Yield per plant } \\
\hline SSR6x-GA24 (VI, 3) & QYld.ncl-1.6 & LG1a (60.4) & TA5-NCPGR56 (VI, 4) & QYld.ncl-2.2 & LG2 (33.9) & - & $-1.69[\mathrm{AAE}(\mathrm{III})], 1.2[\mathrm{AAE}(\mathrm{IV})]$ \\
\hline \multicolumn{8}{|l|}{ 100-seed weight } \\
\hline UBC335y-UBC173 (VI, 4) & QSwt.ncl-1.12 & LG1a (34.6) & TA110-HIB09 (VI, 5) & QSwt.ncl-5.1 & LG5 (38.4) & -2.24 & - \\
\hline \multicolumn{8}{|l|}{ Days to maturity } \\
\hline UBC149z-UBC149x (VI, 2) & QDmt.ncl-1.4 & LG1a (67) & H3A05/2-NCPGR57 (VI, 7) & QDmt.ncl-4.2 & LG4 (35.6) & 0.81 & $-0.88[\mathrm{AAE}(\mathrm{III})], 0.86[\mathrm{AAE}(\mathrm{IV})]$ \\
\hline
\end{tabular}

${ }^{\text {a }}$ In parentheses are population name (JV/VI), followed by marker intervals in Arabic numerals

${ }^{\mathbf{b}}$ AA: additive effect; AAE(I), AAE(II), AAE(III) and AAE(IV): epistasis associated with environments I, II, III and IV, respectively

common in both the populations and expressed in two environments. This QTL was also associated with $Q B r p$. $n c l-1.1$ in the JV population and with QYld.ncl-1.1 and QSwt.ncl-1.7 in the VI population. This association therefore supports the significant positive correlations observed among the traits Pht, Brp, Yld and Swt. Although a total of 28 Psp QTLs were detected (12 in JV and 16 in VI population), there was no common QTL between the populations. It was observed that LG2 in the JV population and LG1 in the VI population contained QTLs for each of the seven traits. Additionally, most of the QTLs detected in the respective population were mapped on these LGs. Hence, concentrating on these LGs in molecular breeding programs could speed up the improvements in these traits.

Three LGs (LG1, 2 and 3) in both the populations shared most of the Brp QTLs. Three Brp QTLs in the JV population were associated with six QTLs for five other traits; QBrp.ncl-1.1 was associated with QPht.ncl-1.1, QBrp.ncl-2.1 was associated with QPsp.ncl-2.1, QPdp.ncl2.1 and QYld.ncl-2.1, while QBrp.ncl-2.2 was associated with QPht.ncl-2.2 and QDmt.ncl-2.1. Similarly, the Psp QTLs were associated with those for Brp and Pdp (LG2 in JV, LG4 in VI), which supports the significant positive correlations observed among these traits in both the populations. QPdp.ncl-2.7 appeared to be pleiotropic with QTLs for Psp, Brp and Yld, whereas QPdp.ncl-3.1 was associated with the QTL QBrp.ncl-3.1 in the JV population. Likewise, $Q P d p . n c l-1.2$ was associated with the trait seeds per pod $(S p p)$ and was also consistent for two years. The Spp was also associated with Yld QTLs. In both the populations, significant correlation was not detected between Yld and Dmt, which could facilitate selection of high-yielding genotypes coupled with early maturity. Similar results have been reported by Kumar and Rao (1996).

In the JV population, only two Swt QTLs were identified on LGs 2 and 4, while as many as 14 Swt QTLs were detected in the VI population. Among the Swt QTLs, QSwt. $n c l-1.4$ was expressed in three environments as well as in the pooled data and was also clustered with the QTLs for Pht and Yld. Cho et al. (2002) first reported a major QTL for Swt, which explained $52 \%$ of the total phenotypic variation. This QTL was also pleiotropic with a QTL for number of seeds per plant. Later, Abbo et al. (2005) also reported three QTLs and Cobos et al. (2007) two QTLs for seed weight. The Swt QTLs identified in the present study 
were also associated with QTLs for the traits Pht, Brp and Yld. Further confirmation of these QTL clusters by addition of more markers and increasing the population size will be useful for improvement of yield and quality in chickpea.

\section{Multiple-trait Composite Interval Mapping (MCIM)}

As many of these agronomic and yield traits were correlated, MCIM and joint MCIM analyses were performed to detect pleiotropic QTLs. Four pleiotropic QTLs in the JV population and three pleiotropic QTLs in the VI population were identified. However, whether the pleotropism is true or due to co-localization of different QTLs needs to be deciphered by fine mapping. In fact, earlier studies have provided evidence for co-localization of QTLs for morphological traits in Arabidopsis (Perez-Perez et al. 2002) and clover (Cogan et al. 2006). Hence, fine mapping could reveal the true nature of pleotropism observed in the present study.

\section{Two-locus analysis}

It is a well known fact that interactions among different loci or QTLs and environment make a substantial contribution to variation in complex traits. A majority of the previous reports on QTL analysis in chickpea did not perform any interaction (QE, QQ and QQE) studies even as QTL analysis with a provision for detecting these interactions would generally avoid the biased estimate of main effect QTLs and increase the success rate in marker assisted selection (MAS) (Kumar et al. 2007). The two locus analysis performed in this study revealed that the QTLs involved in QE interactions were mainly M-QTLs; while the QTLs involved in QQ / QQE interactions were mainly the QTLs, which had no main effects. This suggests that many of these QTLs have no main effects and exercise their effects through interactions with other QTLs, which are either M-QTLs or E-QTLs. Among the 14 M-QTLs, five were involved in $\mathrm{QE}$ interactions (two QE interactions in $\mathrm{JV}$ and three in VI), which were detected in up to three environments. Some of the QTLs were stable across the environments and were not involved in QE interactions. Of the 38 E-QTLs detected, 34 QTLs were involved in either $\mathrm{QQ}$ or QQE interactions. In addition to these QQ interactions, there were three instances (two in JV and one in VI) where the same QTL was involved in two epistatic interactions. These interactions appear to be a pleiotropic QTL interacting with one QTL each for two different traits (Fig. 3).

Two QTLs from JV population and seven QTLs from VI population detected by single locus analysis also showed interactions with other QTLs detected by two locus analysis. This indicated significant contribution of the QTLs by themselves as well as due to interaction with other QTLs and environments. Normally, selection of QTLs for marker assisted selection is based on phenotypic variance explained by a particular QTL; however, by studying both the main effect as well as interaction effect, the effectiveness of selection increases. For QTLs having individual effects, single locus analysis alone would be sufficient; however, detailed analysis is necessary to elucidate interaction effects. Hence, single and two locus analyses together are more useful than either of the approaches used independently.

Common background markers obtained for the traits like plant height (UBC43z), yield (TA103x), 100-seed weight (TAA170, UBC249x) and days to maturity (NCPGR80, UBC346) shows the stability of the respective QTLs across different methods of analyses. The lack of significant interaction with the environment for the QTLs of 100seed weight clearly indicates the presence of major genes as well as a need for a different method of analysis for the trait. The same explanation seems to be applicable for the trait 'days to maturity' as well. Our study underscores that both M-QTLs as well as interacting QTLs control many of the yield related traits. However, the relative importance of epistasis and environment interactions may vary from trait to trait.

\section{Comparison with previous studies}

The results obtained for different traits in the present study are in conformity with earlier reports. In addition, new QTLs for previously studied traits as well as those for new
Fig. 3 QTL $\times$ QTL interactions each involving three QTLs detected through two locus analysis for two different traits: (a) Psp and Brp in JV population, (b) Brp and Pdp in JV population and (c) for Psp and Pdp in VI population

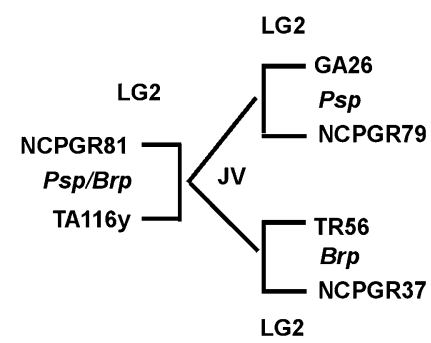

(a)

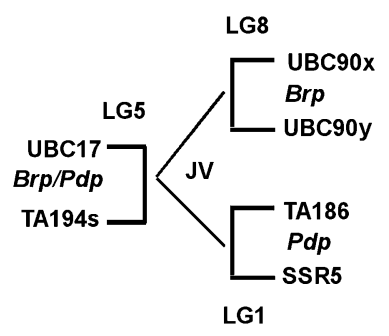

(b)

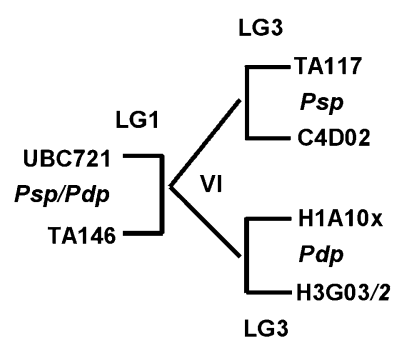

(c) 
traits (Pht, Psp, Brp, Pdp and Dmt) have been identified. Using two populations, up to four environments as well as performing interaction studies enabled us to identify many more QTLs than the simple interval mapping method used in most of the previous studies, which cannot resolve the QTLs involved in different types of interactions. To identify which of these QTLs are main effect QTLs (M-QTLs), or are involved in interactions [QTL $x$ QTL (QQ), QTL $x$ environment (QE) or QTL x QTL x environment (QQE)], we subjected these QTLs to two-locus analysis using QTLNetwork. This analysis identified 14 M-QTLs, 32 E-QTLs and seven pleiotropic QTLs.

A total of 78 QTLs for new traits were identified in both the populations, viz. Pht (11 QTLs), Psp (28 QTLs), Brp (12 QTLs), Pdp (14 QTLs) and Dmt (13 QTLs). Cho et al. (2002) mapped a pleiotropic QTL for seed number per plant and 100-seed weight on LG-IV as well as for 100-seed weight on LG-IX. We identified eight Swt QTLs on LG-1a of VI population, which corresponds to LG-IV of Winter et al. (2000). Hence, it is possible that one or more of our Swt QTLs might be the same as the seed weight QTLs detected by Cho et al. (2002). However, as the marker orders between the map of Cho et al. (2002) and our maps are different, it is difficult to determine the precise correspondence of the QTLs. Similarly, Abbo et al. (2005) detected three seed weight QTLs on LGs I, IV and VI. The "Seed weight QTL 1" of Abbo et al. (2005) was linked to the marker GA24 on LG-IV. We also identified two Swt QTLs (QSwt.ncl-1.1 and QSwt.ncl-1.2) located very close to GA24 on LG1a in the VI map. Hence, either or both of these QTLs might correspond to the "Seed weight QTL 1" of Abbo et al. (2005). Similarly, Cobos et al. (2007) identified three tightly linked QTLs for seed size, yield and days to flowering on LG-IV. In both of our populations, these QTLs were not polymorphic and hence could not be detected, although on the same linkage group (LG1a of VI map, corresponding to LG-IV of Winter et al. 2000), we identified eight Swt QTLs, three Yld QTLs and two Dmt QTLs.

Based on the linked markers, it was interesting to note that LG1 of the JV map and LG1a of the VI map, which correspond to the LGs I, IV and V of the chickpea reference map (Winter et al. 2000), contained QTLs for many traits, viz. plant height, plant spread, number of branches per plant, number of pods per plant, yield, seed weight and days to maturity (this study); seed number per plant, 100seed weight, days to $50 \%$ flowering (Cho et al. 2002); blight resistance (Tekeoglu et al. 2002; Cho et al. 2004; Lichtenzveig et al. 2006); seed weight and lutein concentration (Abbo et al. 2005) and seed size, yield and days to $50 \%$ flowering (Cobos et al. 2007). Hence, concentrating on these LGs in molecular breeding programs might bring in simultaneous improvement in many of these traits.
Potential use of the QTLs in MAS

The development and implementation of MAS for agronomic and yield traits is a high future priority as it will allow identification of relevant recombination events at an early stage without the need for costly and repetitive phenotyping. Co-location of many QTLs in the present study provides confirmation for the genetic basis of correlations and suggests that selection for a small number of target genomic regions may achieve gains for multiple characters. In contrast, the presence of Swt QTLs outside the major clusters observed in the present study may provide the basis for simultaneous and divergent selection of Swt and Yld to improve both seed size/weight and yield. Likewise, inclusion of the QTLs expressed in a particular environment or physiological conditions along with the stable QTLs could be a breeding strategy for developing genotypes adapted to a wide range of environments. The use of two populations and performing interaction analyses in the present study permitted detection of many QTLs, some of which were stable across populations as well as environments. Although only two QTLs (QPht.ncl1.1 and QDmt.ncl-1.1) were common in the two populations, we found common LGs (particularly, LG2 of JV map and LG1a of VI map) for QTLs of many traits. Additionally, QTLs for many other important traits were located on these LGs by previous researchers. Hence, these LGs can be targeted in molecular breeding programs to achieve simultaneous and correlated improvement in these traits. Further, this study also suggests that, for an in-depth genetic dissection of a trait, several diverse mapping populations need to be examined in different environments and locations.

Acknowledgments The financial assistance for this work was provided by the McKnight Foundation, USA. SJMG and PR acknowledge the Council for Scientific and Industrial Research (CSIR), India, for Senior Research Fellowships.

\section{References}

Abbo S, Molina C, Jungmann R, Grusak M, Berkovitch Z, Reifen R, Kahl G, Winter P, Reifen R (2005) Quantitative trait loci governing carotenoid concentration and weight in seeds of chickpea (Cicer arietinum L.). Theor Appl Genet 111:185-195

Cho S, Kumar J, Shultz J, Anupama K, Tefera F, Muehlbauer F (2002) Mapping genes for double podding and other morphological traits in chickpea. Euphytica 128:285-292

Cho S, Chen W, Muehlbauer F (2004) Pathotype-specific genetic factors in chickpea (Cicer arietinum L.) for quantitative resistance to ascochyta blight. Theor Appl Genet 109:733-739

Cobos M, Rubio J, Fernandez-Romero M, Garza R, Moreno M, Millan T, Gil J (2007) Genetic analysis of seed size, yield and days to flowering in a chickpea RIL population derived from a Kabuli x Desi cross. Ann Appl Biol 151:33-42 
Cogan N, Abberton M, Smith K, Kearney G, Marshall A, Williams A, Michaelson-Yeates T, Bowen C, Jones E, Vecchies A, Forster J (2006) Individual and multi-environment combined analyses identify QTLs for morphogenetic and reproductive development traits in white clover (Trifolium repens L.). Theor Appl Genet 112:1401-1415

Deorge R, Churchill G (1996) Permutation tests for multiple loci affecting a quantitative character. Genetics 142:285-294

Eser D (1976) Heritability of some important plant characters, their relationships with plant yield and inheritance of Ascochyta blight resistance in chickpea (Cicer arietinum L.). Ankara Univ Ziraat Faku" ltesi Yayinlari, No. 620, Ankara, Turkey.

IRRI (2005) IRRISTAT for Windows ver. 5.0. - Metro Manila, Philippines.

Kumar J, Rao B (1996) Super-early chickpea developed at the ICRISAT Asia Center. Int Chickpea Pigeonpea Newsl 3:17-18

Kumar N, Kulwal P, Balyan H, Gupta P (2007) QTL mapping for yield and yield contributing traits in two mapping populations of bread wheat. Mol Breed 17:163-177

Lichtenzveig J, Bonfil D, Zhang H, Shtienberg D, Abbo S (2006) Mapping quantitative trait loci in chickpea associated with time to flowering and resistance to Didymella rabiei, the causal agent of Ascochyta blight. Theor Appl Genet 113:1357-1369

Muehlbauer F and Singh K (1987) Genetics of chickpea. In: Saxena $\mathrm{MC}$ and Singh $\mathrm{KB}$ (eds), The chickpea. $\mathrm{CAB}$ International, p. 99-125.
Nelson J (1998) Qgene: Software for maker-based genomic analysis and breeding. Mol Breed 3:239-245

Perez-Perez J, Serrano-Cartagena J, Micol J (2002) Genetic analysis of natural variations in the architecture of Arabidopsis thaliana vegetative leaves. Genetics 162:893-915

Radhika P, Gowda S, Kadoo N, Mhase L, Jamadagni B, Sainani M, Chandra S, Gupta V (2007) Development of an integrated intraspecific map of chickpea (Cicer arietinum L.) using two recombinant inbred line populations. Theor App Genet 115:209216

Singh M (1991) Genotypic and phenotypic correlations in plant traits. Int Center for Agric Res in the Dry Areas, Aleppo, Syria

Tekeoglu M, Rajesh P, Muehlbauer F (2002) Integration of sequence tagged microsatellite sites to the chickpea genetic map. Theor Appl Genet 105:847-854

Voorrips R (2002) MapChart: software for the graphical presentation of linkage maps and QTLs. J Hered 93:77-78

Wang S, Basten C, Zeng Z (2004) Windows QTL Cartographer 2.5. Department of Statistics. North Carolina State University, Raleigh, NC

Winter P, Benko-Iseppon A, Huttel B, Ratnaparkhe M, Tullu A, Sonnante G, Pfaff T, Tekeoglu M, Santra D, Sant V, Rajesh P, Kahl G, Muehlbauer F (2000) A linkage map of chickpea (Cicer arietinum L.) genome based on recombinant inbred lines from a $C$ arietinum $\times C$ reticulatum cross: localization of resistance genes for Fusarium races 4 and 5. Theor Appl Genet 101:1155-1163 